\title{
USO DEL BÓRAX COMO LUBRICANTE PULVIMETALÚRGICO
}

\author{
(Borax as a lubricant in powder metallurgy)
}

\section{Héctor Geovanny Ariza Suarez *, Armando Sarmiento Santos**}

*Maestría en Metalurgia y Ciencia de los Materiales, Uptc. Grupo de investigación GSEC. gseg@uptc.edu.co

**Uptc, grupo de investigación GSEC. gseg@uptc.edu.co

(Recibido: 5 de Agosto de 2014 y aceptado 1 de Octubre de 2014)

\begin{abstract}
Resumen:
En este trabajo se reportan los primeros resultados de la investigación del uso del bórax (di Sodio tetraborato decahidrato) Scharlau ${ }^{\circledR}$, como lubricante pulvimetalúrgico. Se prepararon dos grupos de probetas, en el primer grupo se adicionó el lubricante comercial estearato de zinc y en el segundo grupo el polvo bórax en concentraciones de $0.5 \%$ en peso, usando como matriz el hierro pulvimetalúrgico Ancor Steel $1000^{\circledR}$. El polvo pulvimetalúrgico se mezcló con los lubricantes en un homogenizador rotacional durante 30 minutos, se compactaron las probetas a $700 \mathrm{Mpa}$ en una prensa uniaxial de 15 toneladas. Se realizó un análisis DSC-TGA a la mezcla con bórax. Las probetas se sinterizaron en un reactor de plasma a $1000^{\circ} \mathrm{C}$ durante 30 minutos, con una atmósfera combinada de hidrógeno y argón. Se caracterizó la densidad y la microdureza de las probetas sinterizadas. Se realizó un análisis de DRX para detectar formación de posibles compuestos por la interacción del bórax. Este trabajo muestra que el bórax puede ser utilizado como lubricante en la pulvimetalurgia
\end{abstract}

Palabras clave: lubricante, bórax, pulvimetalurgia, sinterización, Acor Steel 1000, plasma.

\section{INTRODUCCIÓN}

La pulvimetalurgia es una técnica desarrollada para la obtención de piezas en grandes series con un alto grado de calidad y excelentes propiedades de dureza, debido a la distribución homogénea de sus componentes químicos. Entre sus bondades se encuentran el ahorro de tiempo y energía, además permite obtener productos que requieren menor uso de mecanizados para su acabado. Esta tecnología emplea polvos metálicos que se mezclan con diferentes elementos para obtener composiciones químicas precisas, que luego son prensadas en matrices y sinterizadas en hornos, reduciendo la pérdida de materias primas y permitiendo tener un control más detallado de sus características físicas y químicas. Un elemento muy importante en esta técnica y que influye en la densidad de las probetas, es el lubricante (Hwang et al., 1992), el cual es añadido a la mezcla de polvos pulvimetalúrgicos entre 0.5 y $1 \%$ de porcentaje en peso para disminuir la fricción con la matriz en el proceso de compactación, y así poder extraer la probeta compactada.

\section{Abstract:}

This paper reported the first results of the investigation of the use of borax (di sodium tetraborate decahydrate) Scharlau ${ }^{\circledR}$, as a lubricant powder metallurgy. Two groups of samples were prepared by adding in the first group, the commercial lubricant zinc stearate and in the second group the powder borax in concentrations of $0.5 \%$ by weight, using as matrix powder metallurgy Ancor Steel $1000^{\circledR}$ iron. Ancor Steel 1000 was mixed with lubricants in a rotational homogenizer for 30 minutes; the samples were compacted at $700 \mathrm{MPa}$ in a uniaxial press of 15 tons. DSC-TGA analysis of the mixture with borax was realized. The specimens were sintered in a plasma reactor at 1000 for 30 minutes, with a combined atmosphere of hydrogen and argon. Microhardness and density of the sintered samples was characterized. XRD analysis was realized to detect possible compounds formation by interaction of borax. This paper shows that borax can be used as a lubricant in powder metallurgy.

Keywords: lubricant, borax, powder metallurgy, sintering, Ancor Steel 1000, plasma.
Otro procedimiento utilizado para el desmolde es lubricar la matriz electrostáticamente antes de compactar la mezcla de polvos (Yang et al., 2006) y así obtener una mayor densidad. Se podría generar otro estudio sobre la posible aplicación del bórax electrostáticamente. En este trabajo se incorpora el lubricante mezclándolo con polvo pulvimetalúrgico Ancor Steel 1000.

El estearato de zinc es uno de los lubricantes usados en la pulvimetalurgia, se ha utilizado en estudios de polvos de aceros estructurales, añadido en una proporción de $0.9 \%$ (Selecká et al., 2003). Otros lubricantes también han sido utilizados en la pulvimetalurgia, como Acrawax-C, Cera EBS, y el estearato de litio.

En los procesos pulvimetalúrgicos no se registra el uso del bórax como lubricante, sin embargo el polvo de bórax es utilizado en procesos de conformado mecánico como el prensado de láminas de acero, y empleado como lubricante debido a su desempeño a altas presiones (Billigmann, 1979). Además, el bórax se ha añadido a lubricantes naturales para mejorar el desempeño de los mismos (Lovell et al., 2010).
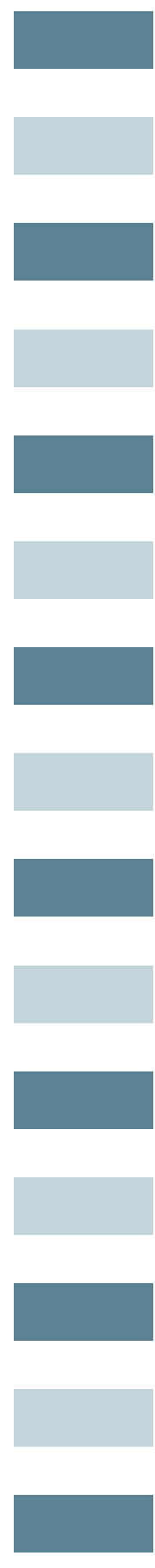
La sinterización de las probetas en verde se ha llevado a cabo en hornos de alto vacío (Yang et al., 2008), tubulares horizontales, microondas (Panda et al., 2006) y de plasma luminiscente (Pavanati et al., 2005; Borgioli et al., 2002). En este proyecto se utiliza un equipo de plasma de descarga luminiscente para la sinterización.

\section{METODOLOGÍA}

Se fabricaron dos grupos de probetas en verde, utilizando como matriz Ancor Steel 1000 suministrado por Hoeganaes Corporation. El Ancor Steel 1000 es un polvo pulvimetalúrgico homogenizado y recocido, producido mediante atomización de acero fundido. Está diseñado para la fabricación de piezas $\mathrm{P} / \mathrm{M}$ (pulvimetalúrgicas) que requieran densidades superiores a $6.7 \mathrm{~g} / \mathrm{cm}$, con características químicas de: $<0.01 \% \mathrm{C},<0.01 \% \mathrm{Si}, 0.2 \% \mathrm{Mn}$, $0.07 \% \mathrm{Cr}, 0.1 \% \mathrm{Cu}, 0.08 \% \mathrm{Ni}, 0.018 \% \mathrm{~S}, 0.009 \% \mathrm{P}, 0.14 \%$ $\mathrm{O}, 0.002 \% \mathrm{~N}$, en peso (Hoeganaes Corporation).

El estearato de zinc y el polvo de bórax se usaron como lubricantes. El estearato de zinc comercial $[\mathrm{CH} 3(\mathrm{CH} 2) 16 \mathrm{COO})] 2 \mathrm{Zn}$, tiene una densidad de 1.095 $\mathrm{g} / \mathrm{cm} 3$ y un punto de fusión alrededor de los $120^{\circ} \mathrm{C}$. Los polvos fueron pesados en una microbalanza Boeco Germany, en una concentración para los lubricantes de $0.5 \%$ en peso. La mezcla de los polvos pulvimetalúrgicos para la elaboración de las probetas en verde se realizó en un homogenizador rotatorio durante 30 minutos. En las tablas 1 y 2 se muestran las características físicas y químicas del bórax.

Tabla 1. Características físicas del bórax

\begin{tabular}{|lc|}
\hline \multicolumn{2}{|c|}{ Datos físicos } \\
\hline Aspecto & Polvo de color blanco \\
Densidad específica & $1.73 \mathrm{~g} / \mathrm{cm} 3$ \\
Punto de fusión & $742^{\circ} \mathrm{C}$ \\
Punto de ebullición & $1575^{\circ} \mathrm{C}$ \\
Presión de vapor & $\left(1200^{\circ} \mathrm{C}\right) 7,3 \mathrm{hPa}$ \\
\hline Fuente: Scharlau ${ }^{\circledR}$ &
\end{tabular}

Tabla 2. Características químicas del bórax

\begin{tabular}{|lr|}
\hline \multicolumn{1}{|c|}{ Análisis } & \multicolumn{1}{c|}{ Valor } \\
\hline Assay (acidimetric) & $100,40 \%$ \\
Identity & passes test \\
Appearance of solution & clear \\
Insoluble in water & $<0,005 \%$ \\
pH $(0,01 \mathrm{M}, \mathrm{H} 2 \mathrm{O})$ & 9,19 \\
Chlorides $(\mathrm{Cl})$ & $<0,001 \%$ \\
Phosphates $(\mathrm{PO} 4)$ & $<0,001 \%$ \\
Sulfates (SO4) & $<0,005 \%$ \\
Ammonium $(\mathrm{NH} 4)$ & $<0,001 \%$ \\
Arsenic (As) & $<0,0005 \%$ \\
Calcium (Ca) & $<0,005 \%$ \\
Copper (Cu) & $<0,0005 \%$ \\
Heavy metals (as $\mathrm{Pb})$ & $<0,001 \%$ \\
Iron (Fe) & $<0,0005 \%$ \\
Lead (Pb) & $<0,0005 \%$ \\
\hline Fuente: Scharlau ${ }^{\circ}$ &
\end{tabular}

Las mezclas de polvos se compactaron a una presión de 700 Mpa en una prensa hidráulica uniaxial de 15 toneladas. Las probetas en verde tienen una geometría cilíndrica de diámetro $10 \mathrm{~mm}$ y altura aproximada de $4 \mathrm{~mm}$. La Figura 1 muestra el reactor de plasma con atmosfera combinada de hidrógeno y argón en el que se sinterizaron las probetas a $1000^{\circ} \mathrm{C}$ durante 30 minutos.

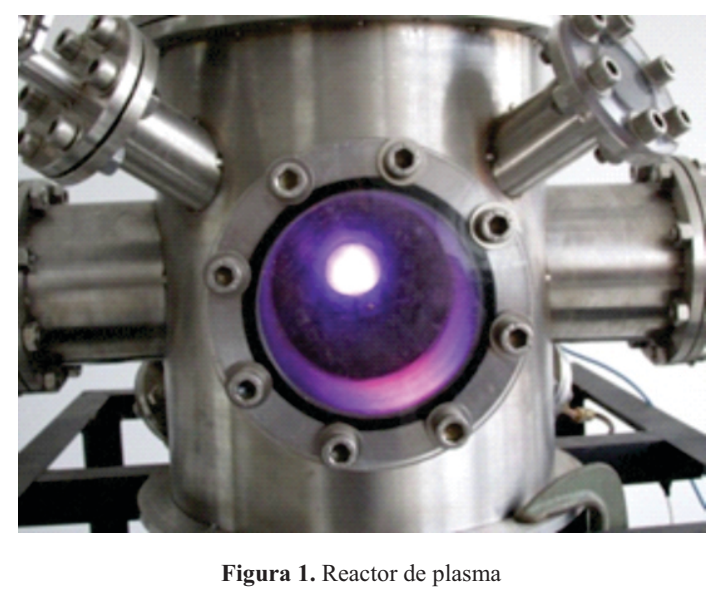

La Figura 2 muestra una de las probetas, bajo la descarga luminiscente, a una temperatura de $1000{ }^{\circ} \mathrm{C}$ con atmosfera combinada $\mathrm{H}+\mathrm{Ar}$.

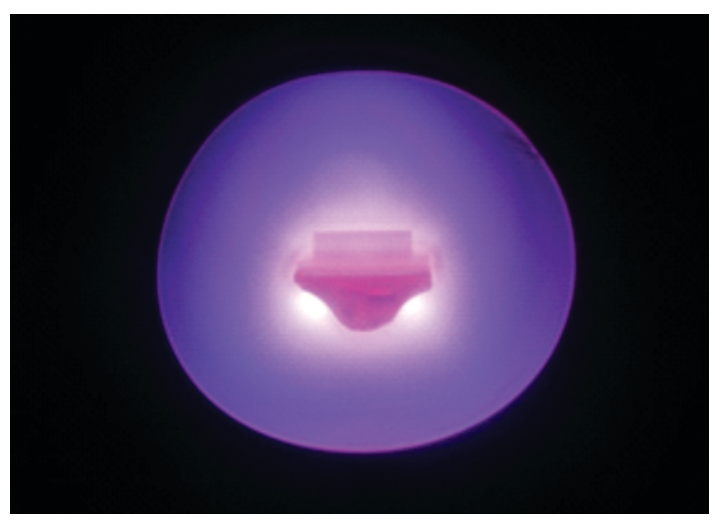

Figura 2. Probeta $0.5 \%$ de bórax bajo una descarga luminiscente

La densidad de las probetas se determinó midiendo su geometría con un calibrador y las masas en una microbalanza electrónica. Se preparó la superficie de las probetas sinterizadas para medir su microdureza iniciando con un desbaste con lijas desde una denominación de 600 hasta una lija 1000.

Para la toma de las micrografías en el microscopio óptico, se prepararon las probetas iniciando con un corte vertical, después fueron puestas en un acrílico autopolimerizable (se ve en la Figura 3) para manipularlas, luego se realiza un desbaste desde una lija número 600 hasta una 2000.

Las probetas montadas en el acrílico se pulieron utilizando alúmina de tamaño $0,3 \mu \mathrm{m}$ en una pulidora metalografía de $200 \mathrm{rpm}$. 


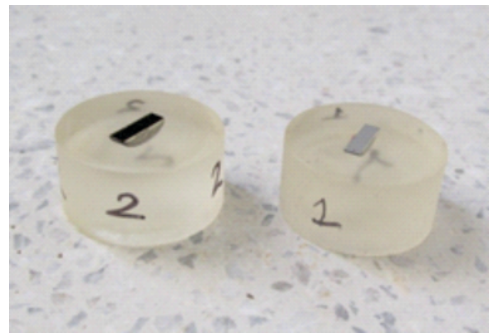

Figura 3. Probetas en acrílico autopolimerizable

La Figura 4 muestra el microdurómetro Qualitest modelo QV-1000 con el cual se midió la microdureza. La huella de una indentación HV0.1 sobre la probeta con $0.5 \%$ de bórax se muestra en la Figura 5.

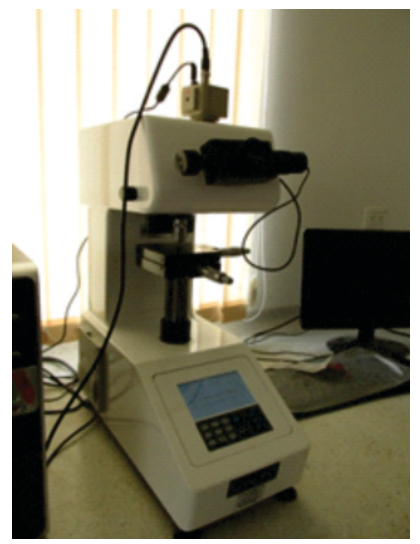

Figura 4. Microdurómetro Qualitest modelo QV-1000

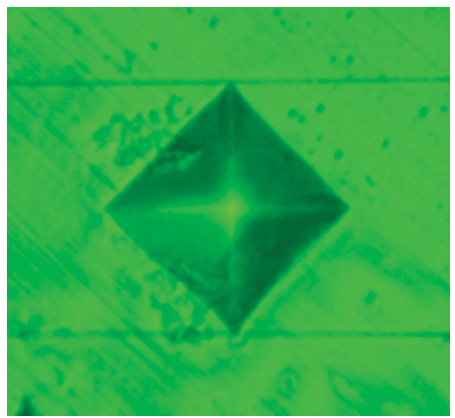

Figura 5. Huella de una indentatación HV0.1

\section{INTERPRETACIÓN DE RESULTADOS}

\subsection{Análisis termogravimétrico}

El análisis DSC-TGA de la mezcla Fe $0.5 \%$ bórax, se muestra en la Figura 6. Este presenta un proceso térmico de deshidratación alrededor de los $143{ }^{\circ} \mathrm{C}$ debido a la presencia del lubricante, en este caso el bórax. El proceso de fusión del bórax, que ocurre alrededor de $\operatorname{los} 743^{\circ} \mathrm{C}$, no es evidente debido a la baja concentración en la muestra.

Las transformaciones alotrópicas del hierro son notorias. La forma alotrópica $\alpha$ (Alfa) con su cambio de parámetro de red se ve alrededor de los $762{ }^{\circ} \mathrm{C}$, y la forma alotrópica $\gamma$ (Gamma), alrededor de los $915.54^{\circ} \mathrm{C}$. Se ve una pérdida de peso después de la deshidratación entre $\operatorname{los} 200^{\circ} \mathrm{C}$ y $500^{\circ} \mathrm{C}$, debida a la salida del lubricante (bórax) de la muestra.
Se observa una ganancia de masa después de los $580^{\circ} \mathrm{C}$, que puede ser debido a la formación de compuestos producto de la interacción con los residuos del bórax, o formación de óxidos por parte del oxígeno atrapado durante la mezcla y compactación de la probeta en verde.

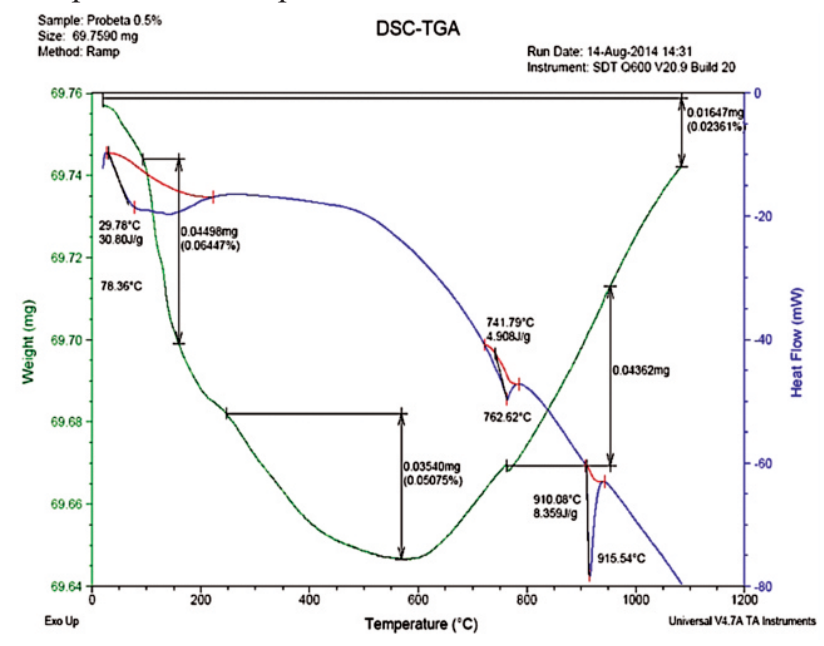

Figura 6. Termogravimetría $0.5 \%$ de bórax

De acuerdo con el analisis de TGA, los residuos del bórax estarian alrededor de un $70 \%$ de los $5 \%$ en peso añadidos a la muestra.

\subsection{Densidad}

La Tabla 3 muestra la densidad de las probetas sinterizadas en el reactor de plasma, para las concentraciones de $0.5 \%$ de bórax y $0.5 \%$ de estearato de zinc. Se observa que las probetas con bórax tienen una densidad similar a las de estearato de zinc.

Tabla 3. Densidad probetas sinterizadas a $1000{ }^{\circ} \mathrm{C}, 30 \mathrm{~min}$.
\begin{tabular}{|l|c|}
\hline \multirow{2}{*}{ Fe $0,5 \%$ bórax } & Densidad $(\mathrm{g} / \mathrm{cm} 3)$ \\
\cline { 2 - 2 } & 6,930 \\
\cline { 2 - 2 } Fe $0,5 \%$ estearato de zinc & 6,867 \\
\hline
\end{tabular}

\subsection{Microdureza}

Los valores de microdureza para las probetas sinterizadas de Ancor Steel 1000, en concentraciones de $0.5 \%$ de bórax y $0.5 \%$ de estearato de zinc, se muestran en la Tabla 4 . Se puede observar que la microdureza obtenida es similar para los dos lubricantes.

Tabla 4. Microdureza probetas sinterizadas a $1000{ }^{\circ} \mathrm{C}, 30 \mathrm{~min}$
\begin{tabular}{|l|c|}
\hline \multirow{2}{*}{ Fe $0,5 \%$ bórax } & Microdureza HV0,1 \\
\cline { 2 - 2 } Fe $0,5 \%$ estearato de zinc & 105,44 \\
\cline { 2 - 2 }
\end{tabular}

\subsection{Análisis de DRX}

La Figura 7 muestra el análisis de DRX realizado a las probetas sinterizadas con $0.5 \%$ de bórax. El ensayo realizado no muestra la formación de compuestos derivados del uso del bórax como lubricante, solo se observan los picos característicos del hierro. 


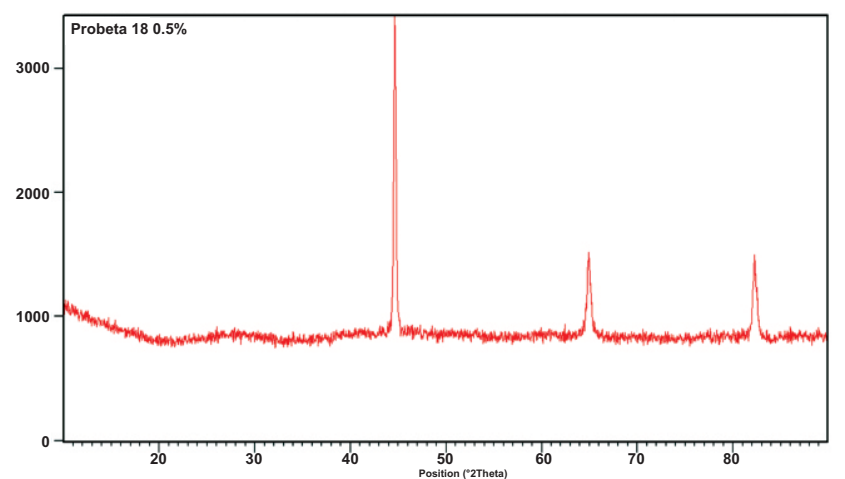

Figura 7. Análisis de DRX probeta sinterizada $0.5 \%$ de bórax

\subsection{Porosidad}

La porosidad es característica de las piezas fabricadas por pulvimetalurgia. En este caso, las probetas con bórax, con una concentración de $0.5 \%$ en peso, presentan una porosidad similar a las probetas con $0.5 \%$ de estearato de zinc, como se ve en las micrografías de las figuras 8 y 9 .
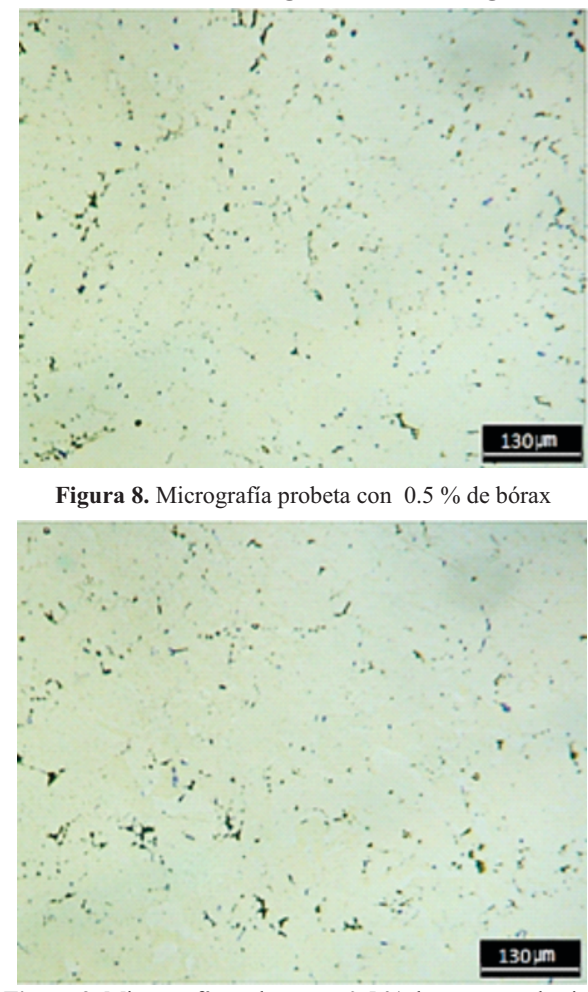

Figura 9. Micrografía probeta con $0.5 \%$ de estearato de zinc

\section{CONCLUSIONES}

El polvo de bórax decahidratado puede ser usado como lubricante pulvimetalúrgico en una concentración de $0.5 \%$ en peso.

Las probetas en verde compactadas a $700 \mathrm{Mpa}$ con $0.5 \%$ de bórax no presentan problemas durante el desmolde.
Los valores de microdureza y densidad de las probetas sinterizadas a $1000{ }^{\circ} \mathrm{C}$ durante 30 minutos, con bórax como lubricante, son similares a los del estearato de zinc a las mismas condiciones. La sinterización del Ancor Steel 1000, con $0.5 \%$ de bórax como lubricante, no muestra formación de compuestos, de acuerdo con el análisis de DRX.

La superficie vista en las micrografías de los dos grupos de probetas presenta una porosidad similar.

\section{AGRADECIMIENTOS}

Agradecemos a Hoeganaes Corporation por suministrarnos el polvo pulvimetalúrgico Ancor Steel 1000.

\section{REFERENCIAS}

Billigmann, J. \& Feldmann, H. (1979). Estampado y prensado a máquina. España: Reverté.

Borgioli, F., Galvanetto, E., Bacci, T. \& Pradelli, G. (2002). Influence of the treatment atmosphere on the characteristics of glow discharge treated sintered stainless steels. Surface and Coatings Technology, 149.

Hwang, K. \& Lin, K. (1992). Lubricant removal in metal powder compacts. International Journal of Powder Metallurgy, 28, 353-360.

Lovell, M., Kabir, M., Menezes, P. \& Higgs III, C. (2010). Influence of boric acid additive size on green lubricant performance. The Royal Society. Rsta, 183.

Panda, S., Singh, V., Upadhyaya, A. \& Agrawal, D. (2006). Sintering response of austenitic (316L) and ferritic $(434 \mathrm{~L})$ stainless steel consolidated in conventional and microwave furnaces. Scripta Materialia, 54.

Pavanati, H., Maliska, A., Klein, A. \& Muzart, J. (2005). Sintering unalloyed iron in abnormal glow discharge with superficial chromium enrichment. Materials Science and Engineering, 392.

Selecká, M., Salak, A. \& Danninger, H. (2003). The effect of boron liquid phase sintering on properties of $\mathrm{Ni}-$, Mo- and $\mathrm{Cr}$-alloyed structural steels. Journal of Materials Processing Technology, 141, 379-384.

Yang, X., Guo, S. \& Akhtar, F. (2006). Lubrication e ffectiveness of composite lubricants during $\mathrm{P} / \mathrm{M}$ electrostatic die wall lubrication and warm compaction. Journal of University of Science and Technology Beijing, Mineral, Metallurgy, Material, 13, 528-531.

Yang, X. \& Guo, S. (2008). Fe-Mo-B Enhanced Sintering of P/M 316L Stainless Steel. Journal of Iron and Steel Research, International. 